\title{
Sobredosificación accidental de vacuna con bacilo de Calmette Guérin
}

\author{
MC. Cubero Sobrados ${ }^{\mathrm{a}}$, C. Sáenz Rodríguez ${ }^{\mathrm{b}}$

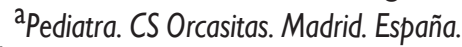 \\ ${ }^{\mathrm{b}}$ Enfermera. CS Imperial. Madrid. España.
}

\section{Resumen}

La vacuna antituberculosa no es una vacuna habitual en los calendarios vacunales españoles, manteniéndose actualmente tan solo en el País Vasco. Dada la escasa frecuencia de vacunación con bacilo de Calmette Guérin en nuestro medio, es raro encontrar casos de sobredosis accidental como el descrito en este artículo.

Palabras clave: bacilo de Calmette Guérin. Tuberculosis. Sobredosificación de fármacos.

Unintentional overdosing of Calmette Guérin vaccination

Abstract

The anti tuberculosis vaccine in Spain is only included in the Basque Country's vaccination schedule. Given the low frequency of vaccination for BCG (Bacille Calmette-Guérin) in our country, it is unusual to find cases of accidental overdose like the one described in this article.

Key words: BCG vaccine. Tuberculosis. Medication Errors.

\section{Introducción}

La vacuna con bacilo de Calmette Guérin (BCG) fue descubierta a principios del siglo xx por Calmette y Guérin, de cuyas iniciales toma el nombre. Fue aplicada por primera vez en 1921 en un recién nacido de madre fallecida por tuberculosis y se inició la vacunación masiva en Francia en 1924, tras la presentación de los primeros resultados vacunales en la Academia de Medicina de París. En España, se puso desde 1927 hasta 1980, manteniéndose en la actualidad en el País Vasco como vacunación a los recién nacidos'.

M. ${ }^{\text {a }}$ Carmen Cubero Sobrados, kr_qb@hotmail.com

Los autores declaran no presentar conflictos de intereses en relación con la preparación y publicación de este artículo. 
Las personas vacunadas desarrollan una respuesta inmunitaria de 8 a 14 semanas después de la vacunación, haciéndose positiva la prueba de la tuberculina (PPD); la negatividad se interpreta como indicador de vacunación no eficaz, aunque esto aún permanece en discusión".

La eficacia, valorada en dos metaanálisis, está en torno al 51\% según distintos ensayos clínicos, y en el $52 \%$ en estudios casos-control (Colditz et al.) $)^{1,2}$.

La reactogenicidad de la vacuna depende en gran medida de la cepa que se utilice y de la técnica de administración utilizada ${ }^{1}$. La complicación más frecuente es la aparición de adenopatía, con supuración o sin ella ${ }^{1-3}$. Las reacciones locales importantes, como úlceras y abscesos, están causadas por una técnica de administración inadecuada, por falta de asepsia, por inyección no intradérmica o por vacunación a individuos con prueba de tuberculina positiva $a^{1,2}$. Cicatrices queloides y reacciones tipo lupus son raras y se han descrito por una técnica de inyección en la zona superior a la inserción del músculo deltoides ${ }^{1}$.

En los niños sanos, inmunocompetentes, las reacciones graves son muy infrecuentes: osteítis (50 casos por millón de vacunados), BCGítis o diseminación generalizada de la vacuna (un caso por millón de vacunados). Encontrándose en es- tos casos algún defecto inmunitario, casi siempre de la inmunidad celular'.

\section{Caso clínico}

Joven de 13 años que acude al centro de salud acompañada de su madre, quien solicita que se le aplique la vacuna antituberculosa. Proyecta un viaje de estudios a Estados Unidos y refieren que es uno de los requisitos para poder acceder a la educación en ciertos centros de ese país. Se le informa de la no disponibilidad de la vacuna en el centro de salud, ya que no es una vacunación presente en el calendario vigente de inmunizaciones de la Comunidad de Madrid, así como de la posibilidad de asistir al Centro de Vacunación Internacional. No obstante, la madre refiere que, por su profesión, tiene acceso a dicha vacuna y solicita tan solo que le sea aplicada en el centro, aportando dicha vacuna la propia familia.

Tras la administración de la vacuna y al quejarse la niña de dolor en el lugar de la inyección, acompañado de sensación de mareo, se procede a atenderla según el protocolo de actuación. Revisando el cartonaje del envase de la vacuna se constata que el vial de $2 \mathrm{ml}$ que se ha inoculado no es monodosis, forma de presentación habitual de las vacunas, sino que contenía 20 dosis, que se inocu- 
laron vía intramuscular, dado el volumen de la presentación (la dosis habitual para adulto es de 0,1 ml, inyectados por vía subcutánea).

Tras comprobar que el estado de la niña es bueno y que presenta constantes vitales normales, se informa a la familia del error cometido y se procede a informar a las autoridades sanitarias pertinentes. Para recabar la mayor información posible sobre la vacuna, nos ponemos en contacto con el Instituto de Toxicología y el Servicio de Medicina Preventiva del Hospital Universitario 12 Octubre, Dirección Gerencia del Área de Referencia, así como con el Servicio de Infecciosas del mismo hospital y con el laboratorio fabricante (Pharmacia Spain) y el Centro de Vacunación Internacional.

Se hizo seguimiento clínico a la niña en el Servicio de Enfermedades Infecciosas del Hospital Universitario 12 de Octubre durante seis meses, realizándose estudios analíticos con pruebas de inmunidad, siendo todas las pruebas normales tanto al inicio del evento como en su evolución. No desarrolló ni en el periodo inmediato ni durante el tiempo de seguimiento ningún tipo de reacción en el lugar de la inyección, por lo que fue dada de alta sin haber presentado incidencia alguna. No recibió ningún tratamiento antituberculoso durante su seguimiento.

\section{Discusión}

Las reacciones adversas a la vacuna BCG están reflejadas para la administración de una dosis y dependen, entre otros aspectos, de la técnica de administración empleada, que debe ser intradérmica. La complicación más frecuente es adenopatía regional, con supuración o sin ella. Otras reacciones como úlceras, abscesos, queloides y lesiones de tipo lupus son menos frecuentes ${ }^{1-4}$.

Las complicaciones más graves son la osteítis/osteomielitis, la infección generalizada por vacuna BCG y la meningitis tuberculosa, generalmente observada en pacientes inmunocomprometidos ${ }^{1,3,4}$.

En Estados Unidos está recomendada la vacuna antituberculosa solamente en casos excepcionales: a) niños con prueba de la tuberculina (PPD) negativa que están expuestos continuamente a pacientes no tratados o ineficazmente tratados, y aquellos expuestos a pacientes con cepas de $M$. tuberculosis resistentes a isoniacida y rifampicina, y b) en ciertas condiciones muy específicas de trabajadores sanitarios con alto riesgo de infección ${ }^{3}$.

El hecho de que la vacuna BCG no sea de uso habitual en nuestro medio explicaría que muchos profesionales sanitarios no tengan experiencia en la administración de la misma, por lo que se producen con más facilidad errores en su administra- 
ción ${ }^{2}$. Desde este punto de vista, es necesario insistir en la mejora del etiquetado de los preparados vacunales, tanto en lo que se refiere a su presentación como a su forma de administración; así como seguir con la tendencia actual de los laboratorios fabricantes en la preparación de las vacunas en formulaciones unidosis. Hemos de poner especial énfasis en la lectura cuidadosa de las fichas técnicas de aquellos productos de escaso manejo en la práctica clínica habitual, con el fin de reducir al mínimo la posibilidad de errores.

El tratamiento antituberculoso en las reacciones adversas vacunales se recomienda para la osteítis y la enfermedad diseminada ${ }^{2-4}$. La mayoría de los expertos no recomiendan el tratamiento de las lesiones cutáneas que se están secando ni de la linfadenitis supurante crónica causada por la vacuna BCG, ya que en la mayoría de los casos se produce resolución espontánea. Las personas con complicaciones causadas por la vacuna BCG deben ser remitidas, si es posible, a un experto en tuberculosis ${ }^{3}$.

En nuestra paciente, dado el buen estado inmunitario y la ausencia de reacciones sistémicas y locales, no fue necesario tratamiento alguno, tan solo el seguimiento aconsejado por un servicio especializado 3 . Por otro lado, aunque según lo publicado se describe que hay mayor ries- go de abscesos y reacciones locales si la inyección no es intradérmica, o bien si es por encima del músculo deltoides ${ }^{1}$; en nuestro caso, en conversaciones mantenidas con los responsables del laboratorio fabricante (Pharmacia Spain) se explicó que el hecho de que la inyección se realizara por vía intramuscular podría haber influido en la no presentación de reacciones locales.

Una última reflexión en lo que se refiere a las demandas a las que nos vemos sometidos como profesionales de la salud diariamente en nuestras consultas: “¿No le va mandar un antibiótico? ¿No me envía a tal o cual especialista?", y un largo etcétera. Cuántas veces tenemos que luchar para no ir en contra de nuestros criterios profesionales, que aplicamos porque creemos correctos, o así lo aprendimos; y cuántas otras veces nos vemos forzados por la presión asistencial, del paciente individual o de sus familiares, a ir en contra de esos mismos criterios. En este caso, no ceder a la insistencia de los padres o familiares, e insistir en nuestro criterio profesional para la vacunación en el Centro de Vacunaciones Internacionales, especializado y más habituado al uso de vacunas fuera del calendario vigente, hubiera evitado tanto a la familia como a nosotros una serie de preocupaciones posteriores. Podemos concluir que aprendimos de ello. 


\section{Bibliografía}

1. Muñiz J. Indicaciones de la vacuna BCG en nuestro medio. En: Campins Martí M, Moraga Llop FA (eds.). Vacunas 2001. Barcelona: Prous Science; 2001. p. 191-204.

2. Campins Martí M, Méndez L, Ramos F, Moreno N, Altet N, Vaqué J. Sobredosis accidental de vacuna BCG. Vacunas. 2003;4:53-5.
3. Committee on Infectious Diseases, American Academy of Pediatrics. Tuberculosis. In: Pickering LK, Baker CJ, Kimberlin DW, Long SS (eds.). Red Book: 2009 Report of the Committee on Infectious Diseases, 28th ed. Elk Grove Village, IL: American Academy of Pediatric; 2009. p. 680-701.

4. Puliyel JM, Hughes A, Chiswick ML, Mughal MZ. Adverse local reactions from accidental BCG overdose in infants. BMJ. 1996;313:528-9. 